\title{
Lung Fibrosis due to Coronavirus Disease 2019 Pneumonia with Critical Symptoms: A Case Report
}

\author{
Bintang Yinke Magdalena Sinaga*(D), Agi Hidjri Tarigan \\ Department of Pulmonology and Respiratory Medicine, Faculty of Medicine, Universitas Sumatera Utara, Adam Malik Hospital, \\ Medan, Indonesia
}

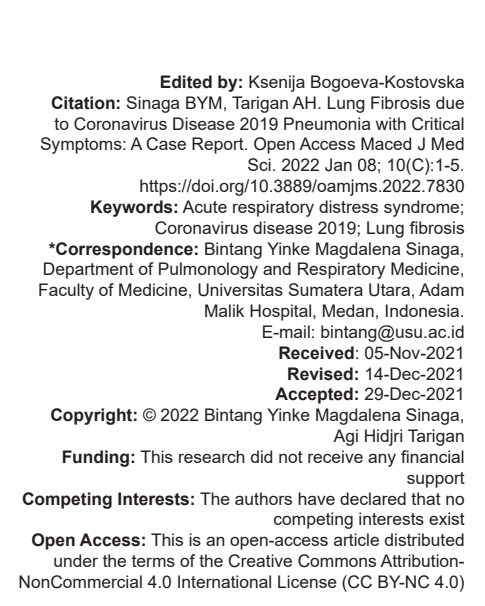

\begin{abstract}
BACKGROUND: The pandemic that occurred at the end of 2019 was caused by the coronavirus 2 (Severe acute respiratory syndrome coronavirus 2 [SARS-CoV-2]). Various speculations mention that the long-term effects of coronavirus disease 2019 (COVID-19) infection can cause pulmonary fibrosis. Acute respiratory distress syndrome (ARDS) is one that can cause pulmonary fibrosis due to injury to the lungs.

CASE REPORT: This report discusses a case of pulmonary fibrosis caused by critical COVID-19 (Coronavirus disease) in 38-year-old male patient with hypertension and obesity comorbidities. The patient was treated for 51 days in intensive care unit with $60 \mathrm{~L} / \mathrm{min}$ high flow nasal cannula assisted oxygenation; then his condition improved as evidenced by his negative Real Time - Polymerase Chain Reaction test result, and was subsequently transferred to a non-COVID-19 ward using non-rebreathing mask at 10-15 L/min, which was later titrated to $2-4 \mathrm{~L} / \mathrm{min}$ nasal canulla. Patient was treated in the non-COVID ward for 16 days. The total number of days of hospitalization was 67 days. Patient had his thorax photo taken 3 times and non-contrast thorax computed tomography (CT) scan 3 times. Based on the evaluation of his thorax CT scan on day 23, we found a vast fibrosis in patient's lungs. Many literatures state that lung fibrosis can be triggered by ARDS, a condition due to the infection from SARS-CoV-2.

CONCLUSION: COVID-19 infection can progress overtime and may cause pulmonary fibrosis. The most serious phase of this virus infection is characterized by sudden and excessive release of proinflammatory mediators that lead to lung damage with large fibrosis and rapid onset of ARDS. To further our understanding of this issue, we present the case report of lung fibrosis caused by critical COVID-19 infection.
\end{abstract}

\section{Introduction}

Novel coronavirus-19 (Severe acute respiratory syndrome coronavirus 2 [SARS-CoV-2]) is responsible for the present pandemic of the coronavirus disease 2019 (COVID-19) which was first reported in Hubei Province, China, on December 31, 2019, and since then has spread all over the world [1]. Data based on early reports from China, which were subsequently proven by more data from Italy, showed that the COVID-19 worst-affected demographic group is elderly male and other bad prognostic factors are history of smoking and comorbidities [2].

Study showed that COVID-19 resulted in bilateral interstitial pneumonia by affecting the tissue covering the lung alveoli. The most serious/critical phase of this virus infection is characterized by sudden and excessive release of proinflammatory mediators that lead to lung damage and rapid onset of acute respiratory distress syndrome (ARDS) with presence of fibrotic tissue due to excess collagen (fibrosis) in the inflamed lung air spaces [3]. It has been found that $40 \%$ of patients with COVID-19 develop ARDS and $20 \%$ of ARDS cases are severe. ARDS itself is one of the most important factors for developing pulmonary fibrosis [4]. Pulmonary fibrosis occurred in $78.9 \%$ of moderate COVID-19 and in all $(100 \%)$ patients with severe or critical COVID-19 [5]. The factors mediating profibrotic response to SARS-CoV-2 virus are not fully known, but some studies suggest that age, severity of illness, use of mechanical ventilation, smoking, and chronic alcoholism may contribute [1].

Pulmonary fibrosis that occurs in COVID-19 patients is different from fibrosis in IPF, where the fibrosis process in IPF occurs progressively [6]. In survivors COVID-19, most patients have shown an improvement or even resolvent of the appearance of fibrosis on chest computed tomography (CT) scans [5], but clinical symptoms may remain even though the patient has recovered, which is also known as postCOVID syndrome [7].

We report a 38-year-old male with critically ill COVID-19 with hypertension and obese comorbid who survived but developed pulmonary fibrosis. The fibrosis improved in 6 months with sequelae symptom. 


\section{Case Report}

A 38-year-old male non-smoker was admitted to emergency room of Haji Adam Malik Central General Hospital, the teaching hospital of Universitas Sumatera Utara in Medan, Indonesia, on September 27, 2020 with main complaint of shortness of breath for 2 days, which worsened 1 day before admission to the hospital. The shortness of breath was not associated with activities, weather, or position. Wheezing and history of wheezing were not found. Unproductive cough started 6 days before admission with no bloody nor history of bloody cough found. The associated chest pain, sore throat, loss of smell, loss of taste, and flu were not present. Neither were nausea, vomiting, and diarrhea (digestive symptoms). Patient had no fever on admission, but reported a history of fever 6 days before with temperature of $38.5^{\circ} \mathrm{C}$ that decreased with antipyretic. Loss of appetite and loss of weight were not found. Hoarseness and swallowing difficulty were not present. History of cephalgia, seizure, and stomach ache was not found. History of antituberculosis treatment and close contact with pulmonary tuberculosis patient was not found. Patient did not have a history of smoking nor asthma and inhaler use. History of allergic such as atopic dermatitis, rhinitis allergic, food, and drugs allergies was not found. Patient was nondiabetic, but had a history of hypertension for 10 years and not well-controlled. He worked as a radiologist at a hospital but maintained no history of close contact with any confirmed/probable COVID-19 cases. History of visiting areas with local transmission of COVID-19 was found in Medan, Indonesia. Previously for the $1^{\text {st }}$ time, the patient experienced shortness of breath and decreased oxygen saturation at $85 \%$ which led him being brought to the hospital.

Vital signs on admission with awareness: Alert, blood pressure: 120/80 mm Hg, Heart rate: 90 times/min, respiratory rate was 31 times/min, and oxygen saturation with non-rebreathing mask (NRM) $15 \mathrm{l} / \mathrm{min}$ was $85 \%$ replaced with high flow nasal cannula (HFNC) $60 \mathrm{l} / \mathrm{min}$ the oxygen saturation become $90 \%$. On physical examination, we found symmetrical chest inspection and there was no lag in breathing. On chest palpation, tactile fremitus was found to be hardened in both lung fields. On percussion examination, both lung fields presented dullness. On auscultation, bronchial breathing sounds and additional crackling sounds were found in both lung fields. Laboratory test results showed increased leukocyte: $17.44 \times 10^{3} / \mathrm{mm}^{3}$, lymphocytopenia: $5.1 \%$, platelet: $348 \times 10^{3} / \mathrm{mm}^{3}$, and hemoglobin $14.6 \mathrm{~g} / \mathrm{dl} \%$. Blood gas analysis indicated type 1 respiratory failure with P/F ratio: 227.5 showed mild ARDS, C-reactive protein quantitative: $400 \mathrm{mg} / \mathrm{dl}$, procalcitonin: $0.4 \mathrm{ng} / \mathrm{ml}$, lactate dehydrogenase (LDH): 401, D-dimer: $2156 \mathrm{ng} / \mathrm{ml}$, ferritin: $2000 \mathrm{ng} / \mathrm{ml}$, total bilirubin: $2.30 \mathrm{mg} / \mathrm{dL}$, SGOT: $67 \mathrm{U} / \mathrm{L}$, SGPT: $63 \mathrm{U} / \mathrm{L}$, ureum: $41 \mathrm{mg} / \mathrm{dL}$, creatinine: $0.55 \mathrm{mg} / \mathrm{dL}$, and increased of lipid profile value. Antibody detection test for
SARS-CoV2 showed immunoglobulin $G$ reactive and the result of Real Time - Polymerase Chain Reaction (RT-PCR) on September 27, 2020 was positive.

The patient had undergone a length of the treatment of 51 days in the intensive care unit (ICU), and the final assessment by RT-PCR in November 16, 2020 was negative, followed by post-COVID care for 16 days in the non-COVID ward. During the treatment, the patient was administered antivirus oseltamivir $2 \times 75 \mathrm{mg}$ for 5 days followed by lopinavir-ritonavir (200 mg/50 mg) two tablets 2 times daily for 10 days, hydroxy chloroquine $2 \times 200 \mathrm{mg}$ for loading dose and 2 $\times 100 \mathrm{mg}$ for day 2-day 7, anti-coagulant enoxaparin 0.6 $\mathrm{cc} / 24 \mathrm{~h}$, corticosteroid methylprednisolone $62.5 \mathrm{mg} / 12 \mathrm{~h}$ with tapering dose, antibiotic meropenem $1 \mathrm{~g} / 8 \mathrm{~h}$ and followed with other antibiotic based on culture, azithromycin $500 \mathrm{mg} / 24 \mathrm{~h}$ for first 7 days, simvastatin $1 \times 20 \mathrm{mg}$, furosemide $20 \mathrm{mg} / 12 \mathrm{~h}$, antihypertension amlodipine $1 \times 10 \mathrm{mg}$, candesartan $1 \times 16 \mathrm{mg}$, bisoprolol $1 \times 2.5 \mathrm{mg}$, Vitamin C $1 \mathrm{~g} / 24 \mathrm{~h}, \mathrm{~N}$-acetylcysteine infusion, and albumin substitution. During patient hospitalization, there was also a secondary infection of bacterial and fungi from sputum culture and the therapy for this seconder infection was given. After 3 days, the blood glucose level was increased so the patient was given rapid-acting insulin (insulin aspartate) $3 \times 6 \mathrm{IU}$ and longacting insulin (insulin glargine) $10 \mathrm{IU}$ at night. Blood tests were carried out routinely and the treatment was adjusted according to the results obtained. The problem of the patient during hospitalization is that for oxygenation the patient still used HFNC for 51 days and the leukocyte increased up to $28.12 \times 10^{3} / \mathrm{mm}^{3}$ and increased of blood glucose level. Supplementation of oxygen used during treatment in the ICU was HFNC, and in non-COVID ward, the patient used NRM titrated to oxygen nasal cannula. During hospitalization, the patient was given medical rehabilitation therapy. Since day 1 of admission to the patient's discharge, the chest $X$-ray was examined 3 times (Figure 1) and CT scan 2 times (Figure 2a and b) to see any changes in the radiological picture.

After discharged from the hospital, the patient did self-rehabilitation in the form of walking and movement exercises which were carried out at home and the patient's strength gradually increased. After 7 months discharged from the hospital, the patient performed chest CT scan and the results showed a decreased of the lung fibrosis, but still present (Figure 2c). The patient's condition was followed up for 1 year, and the main complaint that the patient still fill fatigue and dyspnea with decreased in oxygen saturation.

\section{Discussion}

The pathogenesis of pulmonary fibrosis involves alveolar epithelial damage due to overexpression of 

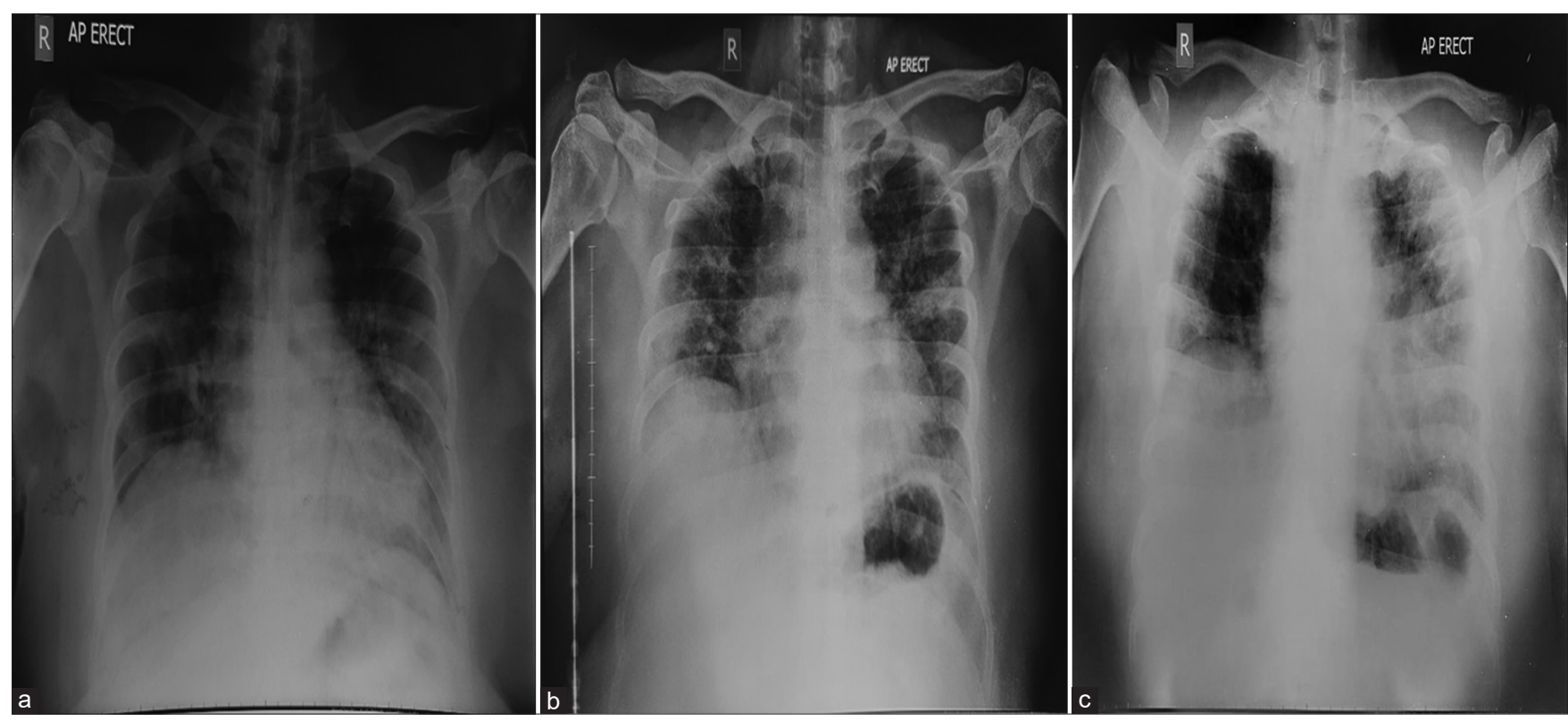

Figure 1: Chest X-rays (a) on September 27, 2020 showed inhomogeneous consolidation in both lung fields with minimal bronchogram. In addition, chest X-rays (b) on November 3, 2020 showed inhomogeneous consolidation in both lung fields with minimal air bronchogram and appeared fibrosis in the right and left lung. The final chest X-rays (c) before discharge on December 2, 2020 showed increased of inhomogeneous consolidation in the left lung appeared fibrosis and in the right lung

proinflammatory cytokines (tumor necrosis factoralpha and interleukins), proliferation and persistence of pro-fibroblastic cells and mediators (fibroblasts, transforming growth factor-beta, fibroblast growth factor, and platelet derived growth factor), and resultant activation of profibrotic pathway. Excess collagen and extracellular matrix replace normal lung tissue and produce architectural distortion typical of interstitial pulmonary fibrosis. Some proposed mechanisms due to fibrosis in COVID-19 include cytokine storm-related hyperimmune response, severe acute lung injury, fibrosing organizing pneumonia, and drug induced and or artificial ventilation-induced lung damage [8], [9]. Risk factors for developing lung fibrosis in COVID-19 patients are old age, comorbidities such as hypertension, diabetes, coronary artery disease, and the presence of lymphopenia, leukocytosis, and increased LDH in laboratory test results. LDH serum level is one of the indicators of the severity of acute lung injury [3] while other study suggests that age, severity of illness, use of mechanical ventilation, smoking, and chronic alcoholism may contribute to lung fibrosis [1]. The patient in this case report was found to have several of the mentioned risk factors, that is, hypertension and obesity comorbidities, abnormal laboratory test results, and a prolonged treatment in ICU with used of HFNC for oxygenation

Although ARDS appears to be a major predictor of fibrosis in COVID-19, several studies showed that COVID-induced ARDS is different from classic ARDS. Chest CT findings in many COVID-19 cases are also not suggestive of classical ARDS. Along with, abnormal coagulopathy is another pathological feature of this
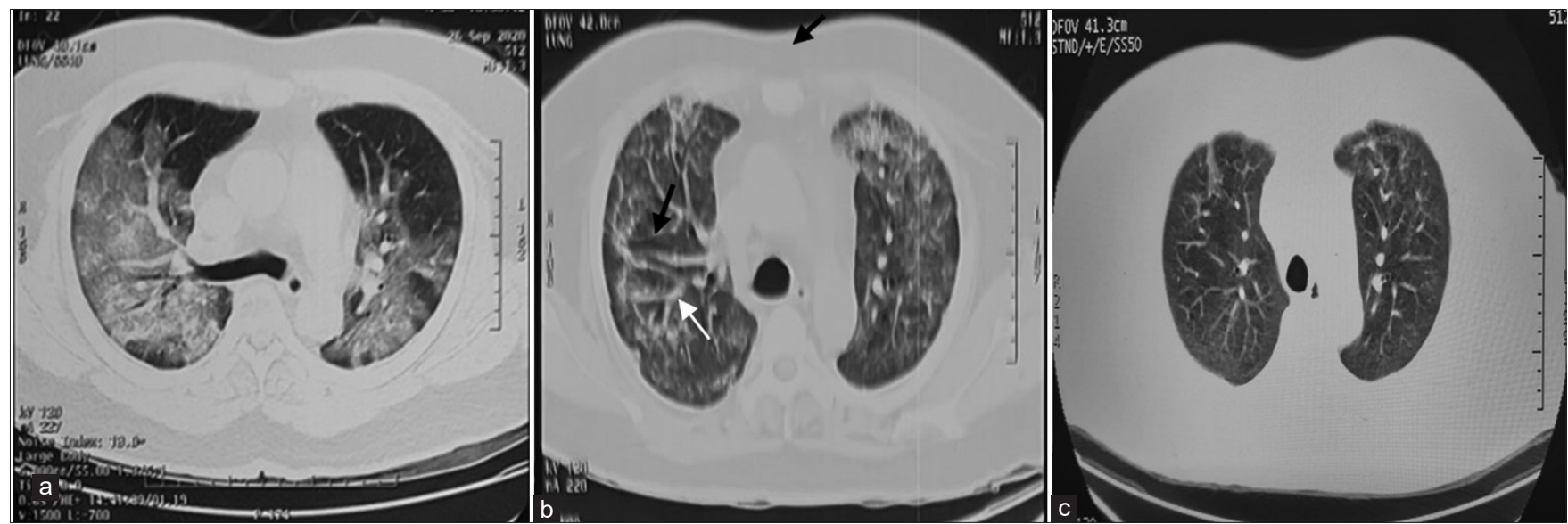

Figure 2: (a) First, the Computed Tomography (CT) Scan lung window was performed on September 26, 2020 showed the ground-glass opacity in both lungs and interstitial thickening bilaterally as compared to, (b) CT scan on November 18, 2020 showed decreased ground-glass opacities in both lung, interstitial thickening (white arrow) and traction bronchiectasis (black arrow) in the right lung. (c) CT scan on July 2 , 2021 showed decreased and improvement of ground glass opacities, interstitial thickening, and traction bronchiectasis compared to Figure $2 b$ 
disease. Hence, mechanism of pulmonary fibrosis in COVID-19 is different from IPF and other fibrotic lung diseases, especially with pathological finding pointing to alveolar epithelial cells being the site of injury, and not the endothelial [4].

The diagnosis of pulmonary fibrosis requires integration of clinical symptoms and radiologic information and history of severe ARDS due to COVID-19. Clinical symptoms of pulmonary fibrosis consisting of dry cough, fatigue, and dyspnea. Lung CT scan finding from pulmonary fibrosis consisting of traction bronchiectasis or bronchiolectasis, honeycombing, or architectural distortion [10]. Fibrosis in COVID-19 patients is unique, because fibrosis can improve completely in one patient while in another it can persist for several months after the patient has recovered. One prospective study was performed to assess long-term lung radiographic changes in patients who recovered from COVID-19 and explore the risk factors for fibrotic-like changes in the lung at 6-month follow-up chest CT of survivors of severe COVID-19 pneumonia. At follow-up CT, evidence of fibrotic-like changes was observed in 40 of the 114 participants $(35 \%)$, whereas remaining 74 participants (65\%) showed either complete radiologic resolution (43 of $114,38 \%$ ) or residual ground-glass opacification or interstitial thickening (31 of 114, 27\%). Multivariable analysis identified age of $>50$ years, heart rate $>100$ beats/min at admission, duration of hospital stay $\geq 17$ days, ARDS, non-invasive mechanical ventilation, and total CT score of 18 or more at initial CT as independent predictors for fibrotic-like changes in the lung at 6 months [11].

In this case report patient, chest CT scan was performed 3 times; at the time of initial symptom onset, after hospitalization for about 60 days and 7 months after the second CT scan. The first CT scan showed extensive ground glass opacity (GGO) with bilateral interstitial thickening, the second CT scan showed interstitial thickening, traction bronchiectasis, and ground glass opacities, while at follow-up 7 months after the patient was discharged from the hospital the interstitial thickening and traction bronchiectasis was reduced, although still present. This patient was admitted to the ICU for 51 days, had ARDS, and had extensive GGO at the time of admission to the hospital.

Functional deficits are also a concern in recovered COVID-19 patients. Several studies have found a decrease in lung function and functional ability accompanied by persistent symptoms known as postCOVID syndrome. In a study of 76 COVID-19 patients (42\% on mechanical ventilation) after 4 months of hospitalization, $53 \%$ had a reduced diffusion capacity, $78 \%$ had a decreased 6 -min walking test, and $53 \%$ had weak grip strength [12]. Another study with 6-month follow-up in 114 patients with severe COVID-19; 6.1\% were still reporting dry cough, $10 \%$ had expectoration, $14 \%$ experienced slight dyspnea on exertion, and $26 \%$ presented with abnormal pulmonary diffusion (DLCO $<80 \%$ predicted) [10]. A multicenter study in 1142 postCOVID-19 patients, fatigue and dyspnea were the main persistent post-COVID-19 symptoms in previously hospitalized patients. Fatigue was present in $61 \%$ patients, dyspnea with activity in $55 \%$, and dyspnea at rest in $23.5 \%$. Only $31.1 \%$ patient did not exhibit fatigue and/or dyspnea 7 months after hospitalization [13]. The patient in this case report, after 1 year of discharge from the hospital, still felt fatigue and shortness of breath with or without activity, but unfortunately, pulmonary function tests were not performed.

To date, there is no specific therapy for postinflammatory pulmonary fibrosis due to COVID-19. Several therapies may be considered to reduce fibrosis due to COVID-19 such as pirfenidone, nintedanib, and mesenchymal stem cells [14], [15], but should be in context with clinical trial. In addition to antifibrotic therapy, respiratory support such as breathing exercise, pulmonary rehabilitation, and pulse oximetry self-monitoring can be performed to reduce complaints due to pulmonary fibrosis and improve quality of life. Indications for specialist assessment include clinical concern along with respiratory, cardiac, or neurological symptoms that are new, persistent, or progressive [16]. The patient in this case report did not carry out pulmonary rehabilitation specifically in the hospital or with professional experts, but the patient did his own exercises at home in the form of walking and other simple movements independently and self-monitoring pulse oximetry. As long as this was done, the patient was working again. After 1 year from the hospital, the clinical symptom was much reduced, but the patient still felt the symptoms of fatigue and shortness of breath even though there was a reduction in intensity over time.

\section{Conclusion}

COVID-19 has progressed over time and caused pulmonary fibrosis. The most serious phase of this virus infection is characterized by sudden and excessive release of proinflammatory mediators that lead to lung damage with rapid onset of ARDS and large fibrosis. Triggers for developing lung fibrosis in COVID-19 patients are old age, comorbidities, the duration of the treatment in ICU and mechanical ventilation, smoking, and high alcohol consumption. Radiology imaging can be describing severity of lung damage and the parenchyma involvement. Several therapies may be considered to reduce fibrosis due to COVID-19. Management of the post-COVID syndrome due to pulmonary fibrosis is a concern to improve the quality of life of post-COVID-19 patients. 


\section{Author's Contribution}

BYMS described and designed the article. BYMS and AHT were involved in treating the patient. BYMS and AHT participated in editing the manuscript critically. All authors declared that they contributed to this article and that they have read and approved the final manuscript.

\section{Acknowledgment}

We thank the health-care workers at Haji Adam Malik General Hospital, Medan, Indonesia, for their assistance in the clinical management of this case.

\section{References}

1. Ojo AS, Balogun SA, Williams OT, Ojo OS. Pulmonary fibrosis in COVID-19 survivors: Predictive factors and risk reduction strategies. Pulm Med. 2020;2020:6175964. https://doi. org/10.1155/2020/6175964

PMid:32850151

2. George PM, Wells AU, Jenkins RG. Pulmonary fibrosis and COVID-19: the potential role for antifibrotic therapy. Lancet Respir Med. 2020;8(8):807-15. https://doi.org/10.1016/ S2213-2600(20)30225-3

PMid:32422178

3. Vitiello A, Pelliccia C, Ferrara F. COVID-19 patients with pulmonary fibrotic tissue: Clinical pharmacological rational of antifibrotic therapy. SN Compr Clin Med. 2020;2(10):1709-12. https://doi.org/10.1007/s42399-020-00487-7 PMid:32875276

4. Rai DK, Sharma P, Kumar R. Post covid 19 pulmonary fibrosis. Is it real threat? Indian J Tuberc. 2021;68(3):330-3. https://doi. org/10.1016/j.ijtb.2020.11.003

PMid:34099197

5. Zou JN, Sun L, Wang BR, Zou Y, Xu S, Ding YJ, et al. The characteristics and evolution of pulmonary fibrosis in COVID19 patients as assessed by Al-assisted chest HRCT. PLoS One. 2020;16(3):e0248957. https://doi.org/10.1371/journal. pone. 0248957

PMid:33755708

6. Selman M, Pardo A. From pulmonary fibrosis to progressive pulmonary fibrosis a lethal pathobiological jump. Am J Physiol. 2020;321:L600-7. https://doi.org/10.1152/ AJPLUNG.00310.2021

PMid:34318695

7. Iqbal FM, Lam K, Sounderajah V, Clarke JM, Ashrafian H, Darzi A. Characteristics and predictors of acute and chronic post-COVID syndrome: A systematic review and meta-analysis. EClinicalMedicine. 2021;36:100899. https://doi.org/10.1016/j. eclinm.2021.100899

8. Nile SH, NileA, Qiu, J, Li L, JiaX, Kai G. COVID-19: Pathogenesis cytokine storm and therapeutic potential of interferons. Cytokine Growth Factor Rev. 2020;53:66-70. https://doi.org/10.1016/j. cytogfr.2020.05.002 PMid:32418715

9. Yuki K, Fujiogi M, Koutsogiannaki S. COVID-19 pathophysiology: A review. Clin Immunol. 2020;215:108427. https://doi. org/10.1016/j.clim.2020.108427 PMid:32325252

10. Solomon JJ, Heyman B, Ko JP, Condos R, Lynch DA CT of post-acute lung complications of COVID-19. Radiology. 2021;301(2):E383-E395. https://doi.org/10.1148/ radiol.2021211396 PMid::34374591

11. Han X, Fan Y, Alwalid O, Li N, Jia X, Yuan M, et al. Six-month follow-up chest CT findings after severe COVID-19 pneumonia. Radiology. 2021;299(1):E177-86. https://doi.org/10.1148/ RADIOL.2021203153

PMid:33497317

12. McGroder CF, Zhang D, Choudhury MA, Salvatore MM, D'Souza BM, Hoffman EA, et al. Pulmonary fibrosis 4 months after COVID-19 is associated with severity of illness and blood leucocyte telomere length. Thorax. 2021;76(12):1242-5. https:// doi.org/10.1136/thoraxjnl-2021-217031 PMid:33927016

13. Fernández-De-Las-Penãs $\quad C, \quad$ Palacios-Cenã $\quad D$ Gómez-Mayordomo V, Palacios-Ceña M, Rodríguez-Jiménez J, de-la-Llave-Rincón $\mathrm{Al}$, et al. Fatigue and dyspnoea as main persistent post-COVID-19 symptoms in previously hospitalized patients: Related functional limitations and disability. Respiration. 2021. https://doi.org/10.1159/000518854

14. Mamun SM A Al, Jahan R, Islam QT, Nazrin T, Shajalal K Rationale of using common antifibrotic therapy in post covid fibrosis. J Med (Bangladesh). 2021;22(1):46-50. https://doi. org/10.3329/jom.v22i1.51391

15. Sequelae L, Bari E, Ferrarotti I, Saracino L, Perteghella S, Torre $\mathrm{ML}$, et al. Mesenchymal stromal cell secretome for post-COVID-19 pulmonary fibrosis: A new therapy to treat the long-term lung sequelae? Cells. 2021;10(5):1203. https://doi. org/10.3390/cells 10051203

PMid:34068958

16. Greenhalgh $T$, Knight $M$, A'Court $C$, Buxton M, Husain L. Management of post-acute covid-19 in primary care. BMJ. 2020;370:m3026. https://doi.org/10.1136/bmj.m3026 PMid:32784198 